Ärztinnen und Ärzte engagieren sich täglich für eine hohe Qualität ihrer Leistungen. Indes verlangen Politik und Öffentlichkeit immer stärker nach Qualitätstransparenz. Mit dem Pilotprojekt Q-Monitoring werden durch Selbstdeklaration Daten über die aktuell betriebene Qualitätssicherung der ambulant tätigen Ärzteschaft der Bereiche Hausarztmedizin und Psychiatrie erhoben.
Die Abteilung Daten, Demographie und Qualität der FMH präsentiert während der Datenerfassung der Pilotphase bis Mitte März 2010 in der Schweizerischen Ärztezeitung drei Interviews mit am Projekt beteiligten Fachpersonen. Sie beantworten darin häufige Fragen im Zusammenhang mit dem Projekt Q-Monitoring.

\title{
Q-Monitoring - Start der Datenerfassung (3) Interview mit Yvette Attinger Andreoli und Hans Kurt
}
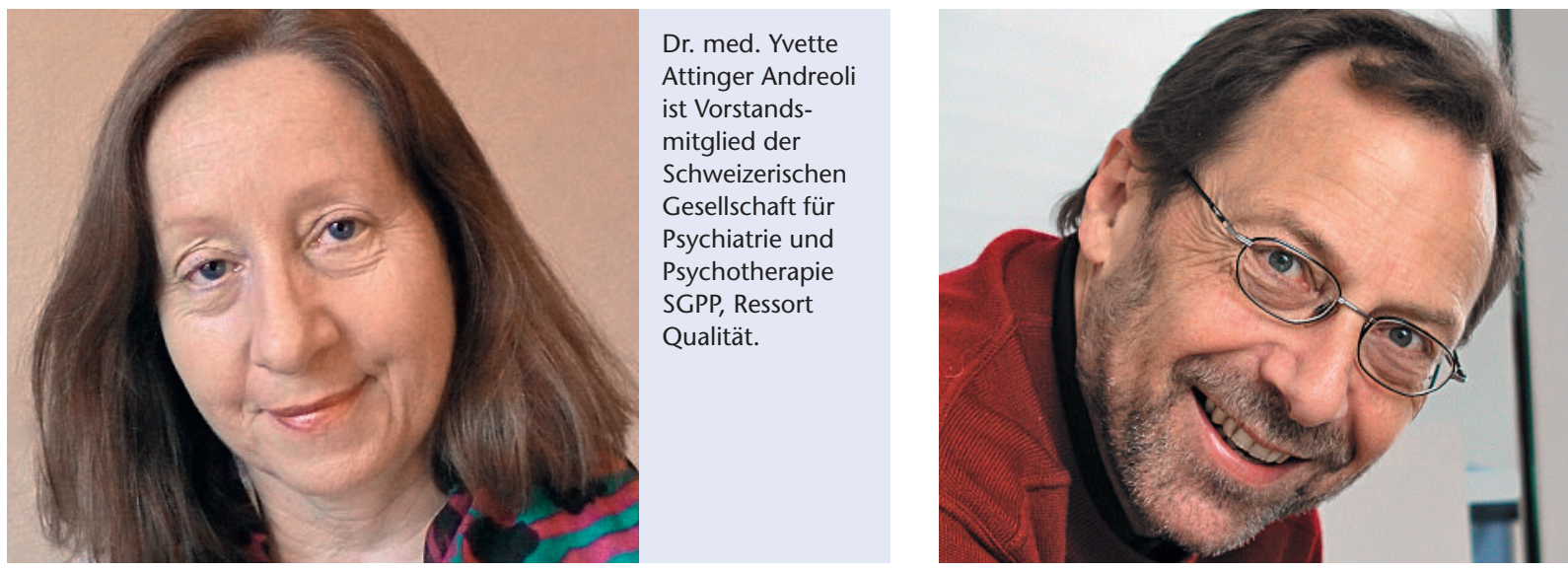

Dr. med. Hans Kurt ist Präsident der SGPP und FMPP (Foederatio Medicorum Psychiatricorum et Psychotherapeuticorum).
Korrespondenz: FMH, Abteilung DDQ Elfenstrasse 18 CH-3000 Bern 15 Tel. 0313591111 Fax 0313591112 ddq@fmh.ch
Wieso engagieren sich die SGPP und die SGKJPP (Schweizerische Gesellschaft für Kinder- und Jugendpsychiatrie und Psychotherapie) im Pilotprojekt Q-Monitoring?

H. Kurt: Qualitätserfassung und -sicherung ist in erster Linie Aufgabe der ärztlichen Fachgesellschaften, denn nur ihnen gelingt eine fachspezifisch sinnvolle und nutzenorientierte Definition von Qualitätsaktivitäten. Durch die Mitarbeit am Projekt Q-Monitoring konnten wir unsere Qualitätsbemühungen intensivieren, uns mit den anderen beteiligten Fachgesellschaften und der FMH austauschen und im Bereich Qualität weiterentwickeln. Im Projekt Q-Monitoring geht es nicht um Kontrolle oder Beeinflussung der psychiatrischen Tätigkeit, sondern darum, unsere bereits bestehenden und erbrachten Leistungen in der Qualität $\mathrm{zu}$ erfassen und gegen aussen sichtbar darstellen zu können.

Wer hat den Fragebogen für das Pilotprojekt Q-Monitoring entwickelt?

Y. Attinger Andreoli: Fachspezifisch zusammengesetzte Teilprojektgruppen haben ein Raster für die Erfassung der spezifischen Qualitätsaktivitäten innerhalb der Fachrichtung erarbeitet. In Zusammenarbeit mit der Abteilung Daten, Demographie und Qualität DDQ der
FMH wurden diese Qualitätsraster zu einem internettauglichen Fragebogen aufbereitet. Dieser lässt sich nun durch die ambulant tätigen Psychiaterinnen und Psychiater auf dem FMH-Mitgliederprofil myFMH elektronisch ausfüllen. Nach dem gleichen Schema entstand in der Teilprojektgruppe Hausarztmedizin ein Fragebogen, der auf die Grundversorger zugeschnitten ist.

Die FMH involviert die Partner im Gesundheitswesen als Beobachter in das Projekt Q-Monitoring. Was halten Sie davon?

H. Kurt: Die SGPP hat schon 2007 ein Zehnpunkte-Positionspapier zur Qualität verabschiedet. Auch gemäss dem Leitbild ärztliche Qualität der FMH ist Qualität eine in Zusammenarbeit mit allen Gesundheitspartnern zu erarbeitende Aufgabe. Deshalb ist es wichtig, möglichst viele dieser Partner in das Projekt Q-Monitoring $\mathrm{zu}$ integrieren. Als Beobachter begleiten das Projekt namentlich das BAG, die GDK, H+, santésuisse, die Stiftung für Patientensicherheit, die Medizinaltarifkommission/SUVA, der Dachverband Schweizerischer Patientenstellen sowie die Krankenversicherungen Helsana, CSS und Concordia. Durch den Einbezug dieser verschiedenen Partner ist ein produk- 
tiver Dialog zum Thema Qualität in der ärztlichen Leistungserbringung für die verschiedenen Interessengruppen entstanden. in dem die eigenen Antworten direkt mit der Gesamtheit der Antworten aus demselben Fachgebiet verglichen werden. Die Auswertung gibt eine Übersicht, in

\section{Fragen, Kritik und Anregungen sind uns sehr willkommen, nur so gelingt es, den Fragebogen und damit die Erfassung der geleisteten Aktivitäten weiter zu verbessern}

Welchen Beitrag können die einzelne Psychiaterin, der einzelne Psychiater resp. die einzelne Kinder- und Jugendpsychiaterin und der einzelne Kinder- und Jugendpsychiater für das Pilotprojekt Q-Monitoring leisten? Und was bringt eine Teilnahme Ihren Mitgliedern?

Y. Attinger Andreoli: Jede Psychiaterin und jeder Psychiater resp. jede(r) einzelne Kinder- und Jugendpsychiater(in) aus dem ambulanten Bereich ist von uns herzlich eingeladen, ihre/seine Qualitätsaktivitäten auf dem Mitgliederprofil myFMH zu deklarieren. Fragen, Kritik und Anregungen sind sehr willkommen, nur so gelingt es uns, den Fragebogen und damit die Erfassung der geleisteten Aktivitäten weiter zu verbessern. Das Ausfüllen des Fragebogens auf myFMH dauert ca. 15 Minuten. Die Teilnehmenden erhalten nach Abschluss der Datenerfassung ein anonymisiertes Profil,

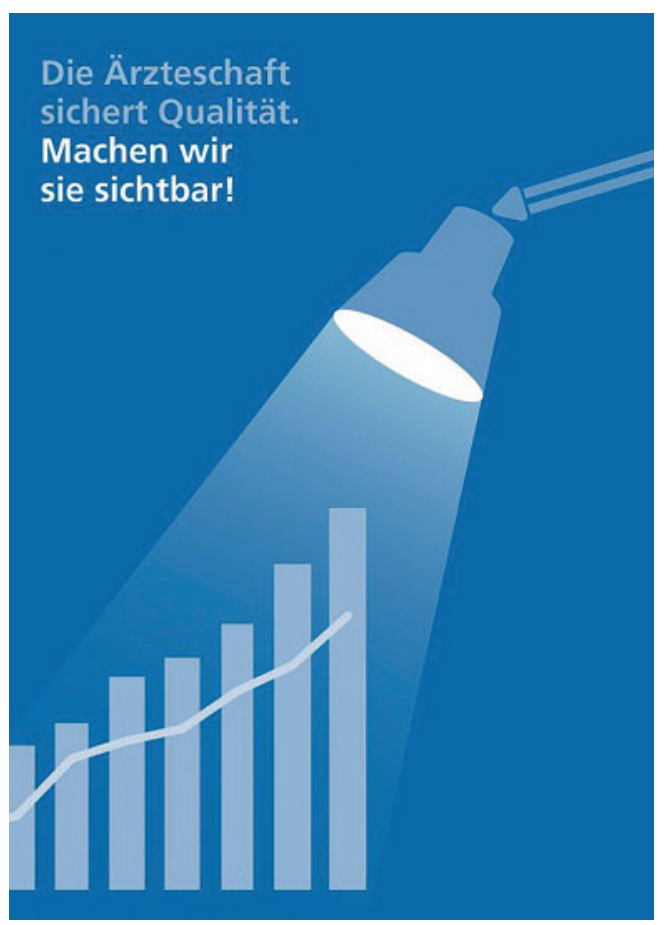

welchen Bereichen bereits viel geleistet wird und wo je nachdem noch Verbesserungen möglich sind. Die Gesamtergebnisse werden zusammen mit Fragen und Kritik anonymisiert an die Fachgesellschaften weitergeleitet. Dies ermöglicht es der SGPP und der SGKJPP, sowohl aussagekräftige Daten für eine sinnvolle Qualitätstransparenz zu gewinnen, als auch besser auf die Bedürfnisse der Psychiaterinnen und Psychiater resp. der Kinder- und Jugendpsychiater einzugehen, sinnvolle Fortbildungen anbieten zu können und eine bessere Vernetzung der Qualitätsbemühungen zu gewährleisten.

\section{Die Datenerfassung läuft noch bis zum 15. März} 2010!

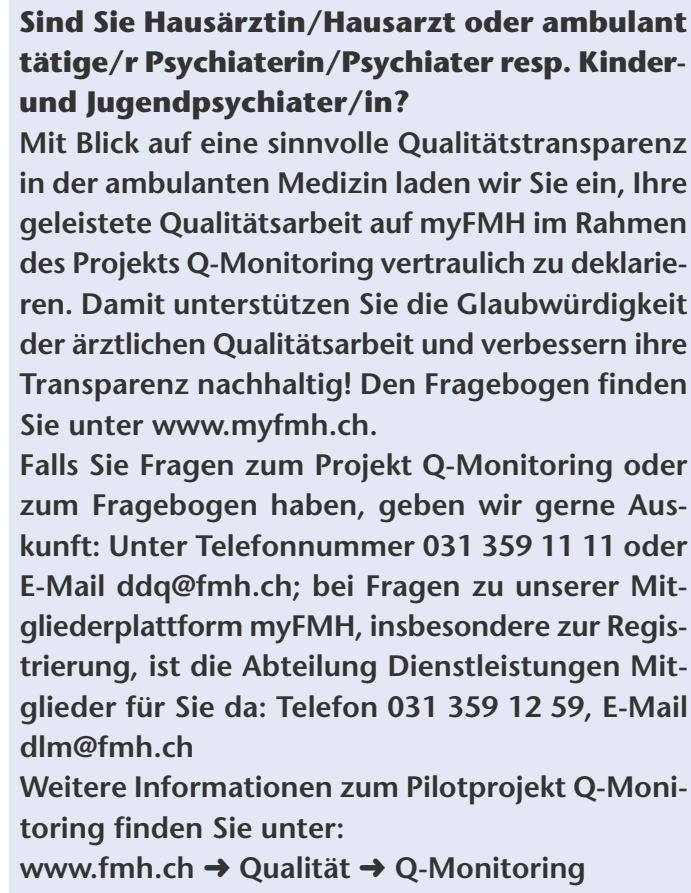

Die ersten beiden Artikel diese Reihe sind in den Ausgaben Nr. 4 und Nr. 6 erschienen. 